\title{
SAVING THE BANDWIDTH IN THE FRACTIONAL DOMAIN BY GENERALIZED HILBERT TRANSFORM PAIR RELATIONS
}

\author{
Soo-Chang Pei, Jian-Jiun Ding \\ Department of Electrical Engineering, National Taiwan University, \\ Taipei, Taiwan, R.O.C \\ Email address:' pei@cc.ee.ntu.edu.tw
}

\begin{abstract}
In this paper, we develop some methods to save the bandwidth required in the fractional domain. The fractional domain is the transformed domain of the fractional Fourier transform (FRFT). It is the intermediate of the time domain and the frequency domain. We find that, with the aid of the fractional Hilbert transform and other techniques, we can save $1 / 2$ or $3 / 4$ of the bandwidth in the fractional domain if the signal is causal, real, a real signal multiplied by chirp, a fractal, or a finite duration signal. The efficiency of the FRFT can hence be improved.
\end{abstract}

\section{INTRODUCTION}

In communication, we usually use the Hilbert transform and other techniques to save the bandwidth in the frequency domain. The Hilbert Transform (HLT) [1] is defined as:

$$
O_{H l}(x(t))=I F T(-j \operatorname{sgn}(\omega) \cdot F T(x(t)))
$$

where FT, IFT are forward / inverse Fourier transforms. If $x(t)$ is causal (i.e., $x(t)=0$ for $t<0$ ), the real part and imaginary part of $X(\omega)(X(\omega)=F T(x(t))$ form a Hilbert transform pair:

$$
\operatorname{Im}(X(\omega))=O_{H l}(\operatorname{Re}(X(\omega))) \quad \text { if } x(t) \text { is causal. }
$$

Since $\operatorname{Im}(X(\omega))$ can be recover from $\operatorname{Re}(X(\omega))$, in the frequency domain we can store $\operatorname{Re}(X(\omega))$ instead of $X(\omega)$, and half of the bandwidth can be saved (because the imaginary part of the spectrum is not required). Besides, the even part and odd part of $X(\omega)$ also form a Hilbert transform pair:

$$
X_{o}(\omega)=O_{H l}\left(X_{e}(\omega)\right) \quad \text { if } x(t) \text { is causal. }
$$

So, in the frequency domain we can also store $X_{e}(\omega) s(\omega)(s(\omega)=$ 0 for $\omega<0, s(0)=1 / 2, s(\omega)=1$ for $\omega>0)$ instead of $X(\omega)$ to save half of the bandwidth (since $X_{e}(\omega) s(\omega)=0$ for $\omega<0$ ).

$$
\begin{aligned}
& \text { Besides, if } x(t) \text { is real, } X(\omega)=F T(x(t)) \text { is conjugate-symmetric: } \\
& X(\omega)=\overline{X(-\omega)} \quad \text { if } x(t) \text { is real }
\end{aligned}
$$

Since $X(-\omega)$ can be recovered from $X(\omega)$ by the above relation, in the frequency domain, we can store $X(\omega) s(\omega)$ instead of $X(\omega)$, and half of the bandwidth can be saved.

Thus, in the frequency domain, we can use the above techniques to save half of the bandwidth if $x(t)$ is causal or real. Then, we may ask whether the same things can be done in the fractional domain. The fractional domain is the transformed domain of the fractional Fourier transform (FRFT) (see equation (5)). Since the FRFT becomes more and more important in signal processing, how to process signal efficiently in the fractional domain also becomes an important topic.
For example, the FRFT have been used for fractional modulation and fractional multiplexing [6], i.e., store and transmit the signal in the fractional domain instead of the frequency domain. Besides, the FRFT can be used for fractional filter and fractional system design [2][6]. There are also many other operations related to the FRFT [2]. They process signals in the fractional domain instead of the frequency domain. If we can develop some techniques to save the bandwidth required in the fractional domain, the efficiency of those operations can all be improved.

In this paper, we develop some methods to save the bandwidth in the fractional domain. We find that, if $x(t)$ is causal, real, a real function multiplied by chirp, a finite duration signal, or a fractal, half of the bandwidth in the fractional domain can be saved. If $x(t)$ is causal-real, a real signal with finite duration, or a real fractal, 3/4 of the bandwidth in the fractional domain can be saved.

First, in Sec. 2, we introduce the FRFT and the fractional Hilbert transform (FRHLT). Then, in Secs. 3 7, we develop some methods to save the bandwidth in the fractional domain. In Sec. 8, we give some examples. In Sec. 9, we give a conclusion.

\section{FRACTIONAL FOURIER / HILBERT TRANSFORMS}

The fractional Fourier transform (FRFT) [2], which is the generalization of the Fourier transform (FT), is defined as

$$
\begin{gathered}
O_{F}^{\alpha}(x(t))=\sqrt{\frac{1-j \cot \alpha}{2 \pi}} \cdot e^{j \cdot \cot \alpha \cdot u^{2} / 2} \int_{-\infty}^{\infty} e^{-j \csc \alpha \cdot u \cdot t} \\
\cdot e^{j \cot \alpha \cdot t^{2} / 2} x(t) \cdot d t \quad \text { when } \alpha \neq N \pi, \\
O_{F}^{2 N \pi}(x(t))=x(t), \quad O_{F}^{2(N+1) \pi}(x(t))=x(-t) .
\end{gathered}
$$

When $\alpha=\pi / 2$, it becomes the FT. It is reversible and additive:

$$
O_{F}^{-\alpha}\left(O_{F}^{\alpha}(x(t))\right)=x(t), O_{F}^{\beta}\left(O_{F}^{\alpha}(x(t))\right)=O_{F}^{\alpha+\beta}(x(t)) .
$$

In this paper, we use $X_{\alpha}(u)$ to denote the FRFT of $x(t)$

$$
X_{\alpha}(u)=O_{F}^{\alpha}(x(t)) \text {. }
$$

The FRFT can extend the utilities of the FT, and is useful for filter design, optical system analysis, pattern recognition, communication, etc. Many signal processing problems that can't be solved well by the original FT will be solved by the FRFT. The FRFT becomes more and more important in signal processing.

The fractional Hilbert transform (FRHLT) [3] is defined based on the FRFT. Its formula is as follows (we generalize the formula in [3] a little): 


$$
\begin{gathered}
O_{H l}^{(a, b), \alpha}(x(t))=O_{F}^{-\alpha}\left(H_{(a, b)}(u) \cdot O_{F}^{\alpha}(x(t))\right) \\
\text { where } \quad H_{(a, b)}(u)=a-j \operatorname{sgn}(u) b .
\end{gathered}
$$

The original HLT, the analytic signal, and the fractional analytic signal [4] are all the special cases of the FRHLT. We will show that the FRHLT is very helpful for saving the bandwidth in the fractional domain (the transformed domain of the FRFT).

\section{REDUCING THE BANDWIDTH OF CAUSAL SIGNALS}

For the case of the FRFT, when $x(t)$ is causal, there is no obvious relation between the real part and odd part of $X_{\alpha}(u)$ (which is the FRFT of $x(t)$ ), and it is hard to find the counterpart of (2). However, we can find the counterpart of (3). If $x(t)$ is causal:

$$
x(t)=0 \quad \text { for } t<0,
$$

in the fractional domain, the even part and the odd part of $X_{\alpha}(u)$ form a fractional Hilbert transform pair (FRHLT pair):

$$
\begin{aligned}
& X_{\alpha, o}(u)=O_{H}^{(0,-j), \pi-\alpha}\left(X_{\alpha, e}(u)\right) \\
& \text { where } X_{\alpha, e}(u)=\left(X_{\alpha}(u)+X_{\alpha}(-u)\right) / 2 \text {, } \\
& X_{\alpha, v}(u)=\left(X_{\alpha}(u)-X_{\alpha}(-u)\right) / 2 \text {. }
\end{aligned}
$$

It is a generalization of (3). Its proof is shown in Appendix.

Thus, if $x(t)$ is causal and in the fractional domain we have known the even part of $X_{\alpha}(u)$, we can use the FRHLT to recover the odd part of $X_{\alpha}(u)$ from (12), and hence the whole value of $X_{\alpha}(u)$. Therefore, in the fractional domain, we only have to store the positive even part of $X_{\alpha}(u)$ :

$$
\tilde{X}_{\alpha, e}(u)=X_{\alpha, e}(u) \cdot s(u)
$$

where $s(u)$ is the step function:

$$
s(u)=0 \text { for } u<0, \quad s(0)=1 / 2, \quad s(u)=1 \text { for } u>0 .
$$

Since $\tilde{X}_{\alpha, e}(u)=0$ for $u<0$, if we store $\tilde{X}_{\alpha, e}(u)$ instead of $X_{\alpha}(u)$, half of the bandwidth can be saved. We can recover $X_{\alpha}(u)$ from $\tilde{X}_{\alpha, e}(u)$ by the following process:

$$
\begin{aligned}
& \text { (1) } X_{\alpha, e}(u)=\tilde{X}_{\alpha, e}(u)+\tilde{X}_{\alpha, e}(-u), \\
& \begin{aligned}
\text { (2) } X_{\alpha}(u) & =X_{\alpha, e}(u)+O_{H}^{(0,-j), \pi-\alpha}\left(X_{\alpha, e}(u)\right) \\
& =O_{H}^{(1,-j), \pi-\alpha}\left(X_{\alpha, e}(u)\right) .
\end{aligned}
\end{aligned}
$$

Similarly, if $x(t)$ is anti-causal:

$$
x(t)=0 \quad \text { for } t>0,
$$

the even part and odd part of $X_{\alpha}(u)$ also form a FRHLT pair:

$$
X_{\alpha, o}(u)=O_{H}^{(0, j), \pi-\alpha}\left(X_{\alpha, e}(u)\right) \text {. }
$$

Notice that the parameters $(0,-j)$ in $(12)$ is changed into $(0, j)$ Thus, in this case we can also store $X_{\alpha, e}(u) \cdot s(u)$ instead of $X_{\alpha}(u)$, and half of the bandwidth in the fractional domain can be saved.

\section{REDUCING THE BANDWIDTH OF REAL SIGNALS}

The FRFT of a real signal does not have conjugate-symmetry property. Thus, the relation as in (4) is no longer satisfied in the fractional domain. However, we can still reduce the bandwidth in the fractional domain with a more complicated method. If $x(t)$ is real, in the fractional domain we can store $A_{\alpha}(u)$ defined as follows instead of $X_{\alpha}(u)$

$$
\begin{aligned}
& A_{\alpha}(u)=\operatorname{Re}\left[\sqrt{j e^{-j \alpha}} e^{-j \cot \alpha \cdot u^{2} / 2} \cdot \tilde{X}_{\alpha, e}(u)\right] \\
& +j \cdot \operatorname{Im}\left[\sqrt{j e^{-j \alpha}} e^{-j \cot \alpha \cdot u^{2} / 2} \cdot \tilde{X}_{\alpha, o}(u)\right] \\
& \tilde{X}_{\alpha, e}(u)=X_{\alpha, e}(u) s(u), \tilde{X}_{\alpha, o}(u)=X_{\alpha, o}(u) s(u) .
\end{aligned}
$$

$X_{\alpha, e}(u), X_{\alpha, o}(u)$ are the even part and odd part of $X_{\alpha}(u)$, and $s(u)$ is defined as in (15). Because

$$
A_{\alpha}(u)=0 \quad \text { for } u<0 \text {, }
$$

half of the bandwidth in the fractional domain can be saved. We can recover $X_{\alpha}(u)$ from $A_{\alpha}(u)$ by

$$
\text { (1) } \begin{aligned}
Z_{\alpha}(u)= & A_{\alpha}(u)+\overline{A_{\alpha}(-u)}, \\
\text { (2) } X_{\alpha}(u)= & \sqrt{-j e^{j \alpha}} \cdot e^{j \cot \alpha \cdot u^{2} / 2} \cdot\left\{Z_{\alpha}(u)+\right. \\
& \left.F T\left[j \tan \left(\sin (2 \alpha) t^{2} / 4\right) \cdot \operatorname{IFT}\left(Z_{\alpha}(u)\right)\right]\right\} .
\end{aligned}
$$

In fact, in (23), $Z_{\alpha}(u)$ is equal to

$$
\begin{aligned}
Z_{\alpha}(u) & =\operatorname{Re}\left(\sqrt{j e^{-j \alpha}} \cdot e^{-j \cot \alpha \cdot u^{2} / 2} X_{\alpha, e}(u)\right) \\
& +j \cdot \operatorname{Im}\left(\sqrt{j e^{-j \alpha}} \cdot e^{-j \cot \alpha \cdot u^{2} / 2} X_{\alpha, o}(u)\right) .
\end{aligned}
$$

The proof of (24) is rather complicated, and can be seen from our manuscript.

Similarly, if $x(t)$ is pure imaginary, we also only have to store the value of $A_{\alpha}(u)$, and half of the bandwidth in the fractional domain can be saved. We can recover $X_{\alpha}(u)$ from (23) and

$$
\text { (2) } \begin{aligned}
X_{\alpha}(u) & =\sqrt{-j e^{j \alpha}} \cdot e^{j \cot \alpha \cdot u^{2} / 2} \cdot\left\{Z_{\alpha}(u)-\right. \\
& \left.F T\left[j \cot \left(\sin (2 \alpha) t^{2} / 4\right) \cdot \operatorname{IFT}\left(Z_{\alpha}(u)\right)\right\}\right\} .
\end{aligned}
$$

\section{REDUCING THE BANDWIDTH OF REAL SIGNALS MULTIPLIED BY CHIRP}

The results in Sec. 4 can be further generalized. If $x(t)$ is a real signal multiplied by chirp:

$$
x(t)=e^{j \cdot \tau \cdot t^{2}} y(t) \quad \text { where } y(t) \text { is a real function, }
$$

and $\tau$ is any real number, then in the fractional domain, we also only have to store the value of $A_{\alpha}(u)$ defined as in (20), and half of the bandwidth in the fractional domain can be saved. We can follow the process similar to (23) and (24) to recover $X_{\alpha}(u)$ from $A_{\alpha}(u)$, except for that (24) is modified as

$$
\text { (2) } \begin{aligned}
X_{\alpha}(u) & =\sqrt{-j e^{j \alpha}} \cdot e^{j \cot \alpha \cdot u^{2} / 2} \cdot\left\{Z_{\alpha}(u)+\right. \\
& \left.F T\left[j \tan \left((\sin (2 \alpha)+\eta) t^{2} / 4\right) \cdot \operatorname{IFT}\left(Z_{\alpha}(u)\right)\right\}\right\}
\end{aligned}
$$

That is, $\sin (2 \alpha)$ is changed into $\sin (2 \alpha)+\eta$.

\section{REDUCING THE BANDWIDTH OF THE SIGNALS WITH FINITE DURATION}

In the case where $x(t)$ is a finite duration signal: 
$x(t) \neq 0$

only when $t \in\left[t_{1}, t_{2}\right]$,

we can use the FRHLT pair relation to save half of the bandwidth required in the fractional domain. In this case, we only have to store the value of $B_{\alpha}(u)$ the fractional domain

$$
B_{\alpha}(u)=\left[\left.e^{j t_{1} \sin \alpha \cdot u} X_{\alpha}\left(u+t_{1} \cos \alpha\right)\right|_{\text {even }} s(u)\right.
$$

where $[f(u)]_{\text {even }}=[f(u)+f(-u)] / 2$. Since $B_{\alpha}(u)=0$ for $u<0$, so half of the bandwidth in the fractional domain can be saved. We can recover $X_{\alpha}(u)$ from $B_{\alpha}(u)$ by the following process:

$$
\begin{aligned}
& \text { (1) } B_{1, \alpha}(u)=\left(B_{\alpha}(u)+B_{\alpha}(-u)\right) / 2 . \\
& \text { (2) } B_{2, \alpha}(u)=O_{H}^{(0,-j), \pi-\alpha}\left(B_{1, \alpha}(u)\right)+B_{1, \alpha}(u) . \\
& \text { (3) } X_{\alpha}(u)=e^{-j t_{1} \sin \alpha\left(u-t_{1} \cos \alpha\right)} B_{2, \alpha}\left(u-t_{1} \cos \alpha\right) .
\end{aligned}
$$

The above results can be proved from the fact that the even and odd parts of $\exp \left(j \cdot t_{1} \sin \alpha \cdot u\right) \cdot X_{\alpha}\left(u+t_{1} \cos \alpha\right)$ form a FRHLT pair:

$$
\begin{aligned}
& O_{H}^{(0,-j), \pi-\alpha}\left\{\left[e^{j t_{1} \sin \alpha \cdot u} X_{\alpha}\left(u+t_{1} \cos \alpha\right)\right]_{\text {even }}\right\} \\
= & {\left[e^{j t_{1} \sin \alpha \cdot u} X_{\alpha}\left(u+t_{1} \cos \alpha\right)\right\rfloor_{o d d} . \text { if } x(t)=0 \text { for } t \notin\left[t_{1}, t_{2}\right] }
\end{aligned}
$$

In signal processing, the signal we deal with is usually a finite duration signal. Using the above method, we can save half of the bandwidth required in the fractional domain.

Some signals are not finite duration signals, but, with a little modification, we can also use the above method to save the bandwidth required in the fractional domain. For example, for a scaling invariant signal (i.e., a fractal) which satisfies:

$$
x(\sigma \cdot t)=\lambda \cdot x(t) \quad \text { where } t \in(-\infty, \infty),
$$

if we know the value of $y(t)$ where

$$
y(t)=x(t) \text { for } t \in\left[t_{1}, t_{2}\right], t_{1}<0<t_{2},
$$$$
y(t)=0 \quad \text { otherwise, }
$$

we can find all the values of $x(t)$ for $t \in(-\infty, \infty)$ by iterative scaling. Thus, we can first convert the fractal $x(t)$ into the finite duration signal $y(t)$, then apply the method described in (30) (33) to save half of the bandwidth in the fractional domain.

\section{THE CASES WHERE WE CAN SAVE 3/4 OF THE BANDWIDTH}

In Secs. 3 6, we have described several conditions where we can save half of the bandwidth in the fractional domain. In fact, sometimes we can further reduce the bandwidth.

If $x(t)$ is real and causal, in the fractional domain, we only have to store the value of

$$
C_{\alpha}(u)=\operatorname{Re}\left(\sqrt{j e^{-j \alpha}} \cdot e^{-j \cot \alpha \cdot u^{2} / 2} \tilde{X}_{\alpha, e}(u)\right)
$$

where $\tilde{X}_{\alpha, e}(u)$ is defined as in (21). Since $\tilde{X}_{\alpha, e}(u)=0$ for $u<$ 0 , so $C_{\alpha}(u)$ is a positive real function. If we store $C_{\alpha}(u)$ instead of $X_{\alpha}(u), 3 / 4$ of the bandwidth in the fractional domain can be saved (since $C_{\alpha}(u)$ has no negative part and positive-imaginary part). We can recover $X_{\alpha}(u)$ from $C_{\alpha}(u)$ by the following process, which is in fact the combination of (23, (24), and (17):

$$
\begin{aligned}
& \text { (1) } \begin{aligned}
Z_{\alpha}(u)= & C_{\alpha}(u)+C_{\alpha}(-u), \\
\text { (2) } X_{\alpha, e}(u)= & \sqrt{-j e^{j \alpha}} \cdot e^{j \cot \alpha \cdot u^{2} / 2} \cdot\left\{Z_{\alpha}(u)+\right. \\
& \left.F T \mid j \tan \left(\sin (2 \alpha) t^{2} / 4\right) \cdot \operatorname{IFT}\left(Z_{\alpha}(u)\right)\right\},
\end{aligned} \\
& \text { (3) } X_{\alpha}(u)= O_{H}^{(1,-j), \pi-\alpha}\left(X_{\alpha, e}(u)\right) .
\end{aligned}
$$

Similarly, if $x(t)$ is real and anti-causal, or pure imaginary and causal (or anti-causal), we can also store $C_{\alpha}(u)$ defined as in (37) instead of $X_{\alpha}(u)$, and save $3 / 4$ of the bandwidth in the fractional domain. We can also use the above process with a little modification to recover $X_{\alpha}(u)$ from $C_{\alpha}(u)$. If $x(t)$ is pure imaginary and causal (or anti-causal), the $2^{\text {nd }}$ step is modified as:

$$
\text { (2) } \begin{aligned}
X_{\alpha, e}(u)=\sqrt{-j e^{j \alpha}} \cdot e^{j \cot \alpha \cdot u^{2} / 2} \cdot\left\{Z_{\alpha}(u)-\right. \\
\left.F T \mid j \cot \left(\sin (2 \alpha) t^{2} / 4\right) \cdot \operatorname{IFT}\left(Z_{\alpha}(u)\right)\right\} .
\end{aligned}
$$

If $x(t)$ is real (or pure imaginary) and anti-causal, the $3^{\text {rd }}$ step is modified as

$$
\text { (3) } X_{\alpha}(u)=O_{H}^{(1, j), \pi-\alpha}\left(X_{\alpha, e}(u)\right) \text {. }
$$

Moreover, if $x(t)$ is a real (or pure imaginary) signal with finite duration, we only have to store the value of $D_{\alpha}(u)$ defined as follows to save $3 / 4$ of the bandwidth in the fractional domain.

$$
D_{\alpha}(u)=\operatorname{Re}\left[\sqrt{j e^{-j \alpha}} e^{j \sin (2 \alpha) t_{1}^{2} / 4} e^{-j \cot \alpha \cdot u^{2} / 2} B_{\alpha}(u)\right] s(u)(43)
$$

where $B_{\alpha}(u)$ is defined as in (30).

\section{EXPERIMENTS}

Wee do some experiments to illustrate the concepts introduced in this paper. Fig. 1(a) is a real signal. Its FRFT is shown in Fig. 1(b). It is not band-limited, but we can use $(20) \sim(25)$ to save half of the bandwidth in the fractional domain. Figs. 1(c) and $1(d)$ are

Fig. 1(c): $\exp \left(-j \cot \alpha \cdot u^{2} / 2\right) X_{\alpha, e}(u)$

Fig. 1(d): $\exp \left(-j \cot \alpha \cdot u^{2} / 2\right) X_{\alpha, o}(u)$.

Fig. 1(e) is the combination the real part of Fig. 1(c) and the imaginary part of Fig. 1(d). It is equal to $Z_{\alpha}(u)$ defined in (25). Since $Z_{\alpha}(u)=\operatorname{conj}\left(Z_{\alpha}(-u)\right)$, the negative part of $Z_{\alpha}(u)$ is redundancy. Thus, we can take its positive part, as in Fig. 1(f). It is in fact equal to $A_{\alpha}(u)$ defined in (20). Since $A_{\alpha}(u)=0$ for $u<0$, if we store $A_{\alpha}(u)$ instead of $X_{\alpha}(u)$, half of the spectrum in the fractional domain is saved. We can recover $Z_{\alpha}(u)$ from $A_{\alpha}(u)$ by (23), and use (24) to recover $X_{\alpha}(u)$ from $Z_{\alpha}(u)$.

In Fig. 2, we give another example. The signal in Fig. 2(a) is a real-causal signal. It is not band-limited in the fractional domain, as in Fig. 2(b). However, we can use (37) (40) to save 3/4 of the bandwidth in the fractional domain. We can take the even part of $X_{\alpha}(u)$ (as in Fig. 2(c)), then multiply it by $\left(j e^{-j \alpha}\right)^{1 / 2} e^{-j \cot \alpha u^{2} / 2}$ and take the real part (as in Fig. 2(d)), then take the positive part (as in Fig. 2(f)). The result in Fig. 2(f) is equal to $C_{\alpha}(u)$ defined in (37). Since

$C_{\alpha}(u)=0$ for $u<0, \quad \operatorname{Im}\left(C_{\alpha}(u)\right)=0$,

$3 / 4$ of the spectrum in the fractional domain is saved. We can recover $X_{\alpha}(u)$ from $C_{\alpha}(u)$ by (38), (39), and (40). The outputs of Steps 1, 2, 3 are just Figs. 2(d), 2(c), and 2(b), respectively. 
(a) a real signal $x(t)$

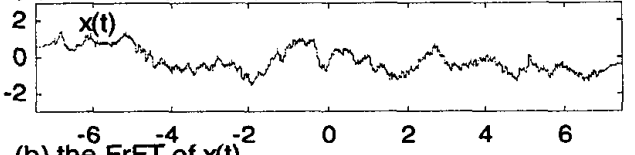

(b) the $\mathrm{FrFT}^{-4}$ of $x(\mathrm{t})^{-2}$

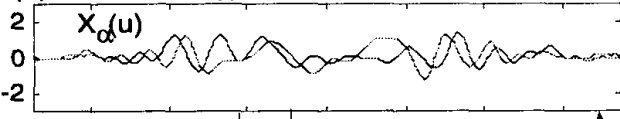

(c) take even part fand multipliêd by chirp ${ }^{-6}$

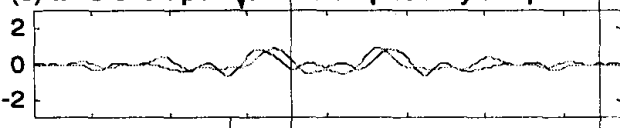

(d) take odd part and multipliêd by chirp ${ }^{-2}$ recover

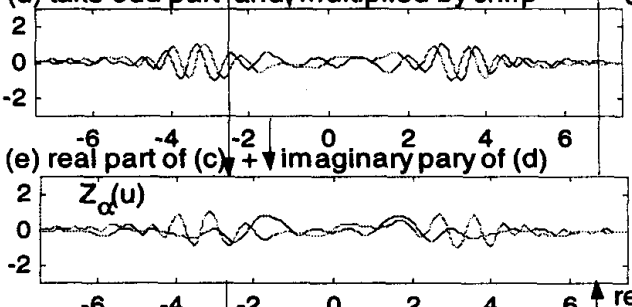
Step 2
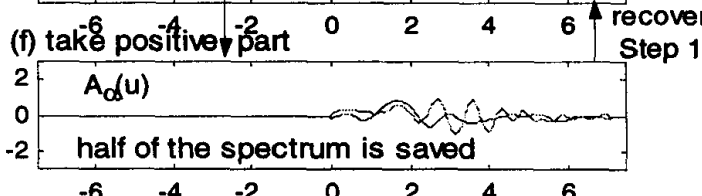

Fig. 1 Saving bandwidth in the fractional domain for a real signal. We use two lines to represent real and imaginary parts.

(a) a real, causal signal $x(t)$
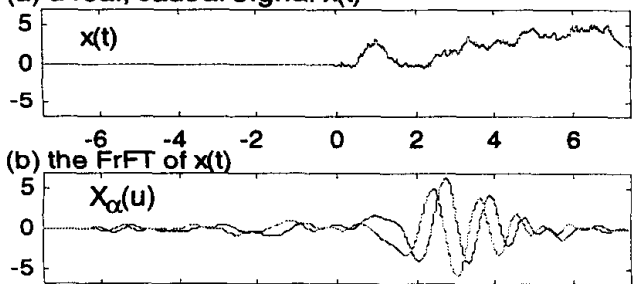

(c) $\operatorname{take}^{-6}$ even $^{-4}$ part $^{-2}$ of $t x_{\alpha}^{0}(u) \quad 2 \quad 4 \quad 6$ recove

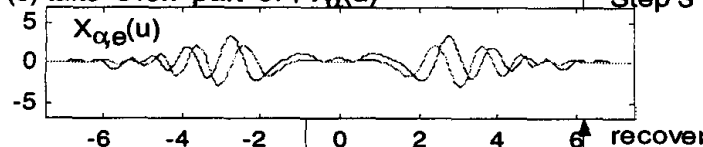

(d) multiplied by chirpsand take real part Step 2
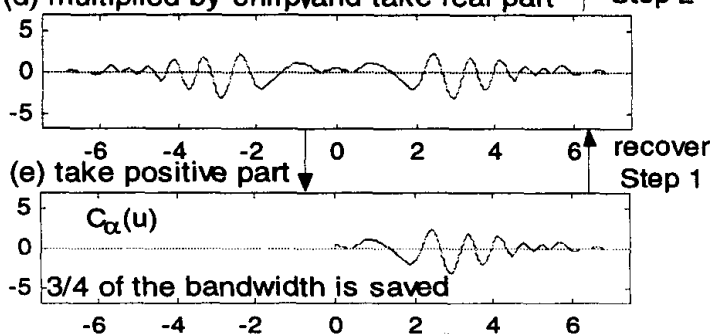

Fig. 2 Saving bandwidth in the fractional domain for a real and causal signal.

\section{CONCLUSION}

In this paper, we have introduced some methods to reduce the bandwidth required in the fractional domain (the transformed domain of the fractional Fourier transform (FRFT)). We find that:

- In following conditions, we can save half of the bandwidth in the fractional domain:

(1) real (or pure imaginary), (2) causal (or anti-causal),

(3) real function $\times$ chirp, (4) finite duration, (5) fractal.

- In following conditions, we can save $3 / 4$ of the bandwidth in the fractional domain:

(1) real (or pure imaginary) \& causal (or anti-causal),

(2) real (or pure imaginary) \& finite duration (or a fractal).

Thus, by the methods introduced in this paper, we can improve the efficiency of the FRFT in signal processing.

\section{REFERENCES}

[1] S. C. Hahn, "Hilbert Transforms in Signal Processing", Boston: Artect House, 1996.

[2] H. M. Ozaktas, M. A. Kutay, and Z. Zalevsky, "The Fractional Fourier Transform with Applications in Optics and Signal Processing", John Wiley \& Sons, 2000.

[3] A. W. Lohmann, D. Mendlovic, and Z. Zalevsky, 'Fractional Hilbert transform', Opt. Lett., vol. 21, no. 5, p 281 283, Feb. 1996.

[4] A. I. Zayed, 'Hilbert transform associated with the fractional Fourier transform', IEEE Signal Processing Lett., vol. 5, no. 8, p 206-208, Aug. 1998.

[5] S. C. Pei and M. H. Yeh, 'Discrete fractional Hilbert transform', Proc. of IEEE Int'l Symp. on Circuits and Systems, vol. 4, p. 506-509, Jun. 1998.

[6] H. M. Ozaktas, B. Barshan, D. Mendlovic, L. Onural, 'Convolution, filtering, and multiplexing in fractional Fourier domains and their rotation to chirp and wavelet transform' J. Opt. Soc. Am. A, vol. 11, no. 2, p. 547-559, Feb. 1994.

[7] S. C. Pei and J. J. Ding, 'Bandwidth saving and edge detection by fractional Hilbert transforms', submitted to $I E E E$ Trans. Signal Processing.

\section{APPENDIX}

(Proof of (12)):

If $x_{e}(t), x_{o}(t)$ are the even part and the odd part of $x(t)$, then

$$
X_{\alpha, e}(u)=O_{F}^{\alpha}\left(x_{e}(t)\right), \quad X_{\alpha, o}(u)=O_{F}^{\alpha}\left(x_{o}(t)\right) .
$$

Then, from the additivity property of the FRFT (see (7)), and the fact that $O_{F}{ }^{\pi}(x(t))=x(-t)($ see $(6))$, we obtain

$$
\begin{aligned}
& O_{F}^{\pi-\alpha}\left(X_{\alpha, e}(u)\right)=O_{F}^{\tau}\left(x_{e}(t)\right)=x_{e}(-t)=(x(-t)+x(t)) / 2, \\
& O_{F}^{\pi-\alpha}\left(X_{\alpha, o}(u)\right)=O_{F}^{\tau}\left(x_{o}(t)\right)=x_{o}(-t)=(x(-t)-x(t)) / 2 .
\end{aligned}
$$

Since $x(t)$ is causal, we conclude that $x_{0}(-t)=-\operatorname{sgn}(t) \cdot x_{e}(-t)$. So

$$
O_{F}^{\pi-\alpha}\left(X_{\alpha, o}(u)\right)=-\operatorname{sgn}(t) \cdot O_{F}^{\pi-\alpha}\left(X_{\alpha, e}(u)\right) .
$$

Since $-\operatorname{sgn}(t)=H_{0,-j}(t)$,

$$
X_{\alpha, o}(u)=O_{F}^{\alpha-\pi}\left(H_{0,-j}(t) O_{F}^{\pi-\alpha}\left(X_{\alpha, e}(u)\right)\right)=O_{H}^{(0,-j), \pi-\alpha}\left(X_{\alpha, e}(u)\right) .
$$

